\title{
Lean capacity planning for tool room: An iterative system improvement approach
}

\author{
Haider, A. ${ }^{a}{ }^{,}$, Mirza, J. ${ }^{a}$, Ahmad, W. ${ }^{a}$ \\ ${ }^{a}$ University of Engineering and Technology, Department of Industrial Engineering, Taxila, Pakistan
}

\begin{abstract}
A B S T R A C T
Capacity planning helps to synchronize demands and production volumes, and assists in early preparedness for dealing with future production imbalances. A tool room of armored manufacturing organization was selected for this work study. Initially, a master production schedule and rough cut capacity plans are prepared based on the delivery commitments of the organization. Time and motion study of the tool room for 28 weeks was performed to determine its current production capacity. It was observed that imbalance between demands and production exists. An iterative system improvement strategy was proposed for exploring the potential of tool room. Lean concepts and methodologies were the essence of the proposed system improvement strategy. It was observed that WIP had been reduced by $18 \%$, production volumes had improved by $24 \%$ and tardiness reduced by $28 \%$. This strengthened our belief that lean philosophy is equally applicable within the capacity planning domain. The capacity planning approach presented in this work study could also be generalized for other domains. This approach believes in improving the potential through elimination of wastes, process improvement, defect prevention and total preventative maintenance. This approach improves the system optimally by utilizing existing resources available on the shop floor. This research will be further expanded towards the major production units of this organization. We are currently working on capacity planning for rebuilding the activities of T-80 UD battlefield tanks.
\end{abstract}

\section{ARTICLE INFO}

Keywords:

Capacity planning

Lean thinking

Manufacturing

Tool room

Simulation

*Corresponding author: aftab775@yahoo.com

(Haider, A.)

Article history:

Received 26 February 2015

Revised 3 October 2015

Accepted 20 October 2015

(c) 2015 PEI, University of Maribor. All rights reserved. 


\title{
Vitko planiranje kapacitet za orodjarno: Iterativni pristop izboljševanja sistema
}

\author{
Haider, A. ${ }^{a,}{ }^{,}$, Mirza, J. ${ }^{a}$, Ahmad, W. ${ }^{a}$ \\ ${ }^{a}$ University of Engineering and Technology, Department of Industrial Engineering, Taxila, Pakistan
}

\section{POVZETEK}

Planiranje kapacitet pomaga pri usklajevanju med povpraševanjem in količino izdelkov ter prispeva k uravnavanju morebitne bodoče proizvodne neuravnoteženosti. Za potrebe raziskave smo izbrali podjetje, ki se ukvarja z izdelavo oklepnih vozil. Najprej je bil na osnovi dobavnih obvez podjetja pripravljen glavni proizvodni program in program za planiranje kapacitet. Da bi določili obseg proizvodnje smo izvedli študij dela v orodjarni za časovni interval 28 tednov. Ugotovljena je bila neuravnoteženost med potrebami in proizvodnjo. Da bi raziskali zmožnosti orodjarne smo predlagali strategijo za iterativni pristop izboljševanja sistema. Bistvo predlagane strategije temelji na konceptu in metodologijah vitkosti. Ugotovljeno je bilo, da se je nedokončana proizvodnja (WIP) zmanjšala za $18 \%$, obseg proizvodnje se je izboljšal za $24 \%$, zakasnitve pa so se zmanjšale za $28 \%$. Ta dejstva so okrepila našo prepričanje, da je filozofija vitkosti primerna za uporabo na področju planiranja kapacitet. Pristop planiranja kapacitet, ki je predlagan v pričujočem delu, je splošen in se lahko uporabi tudi na drugih področjih. Ta pristop nakazuje, da se izboljšanje sposobnosti sistema doseže z zmanjšanjem odpadkov, izboljšanjem procesa, preprečevanjem okvar in celostnega preventivnega vzdrževanja. Pristop izboljšuje optimalno delovanje sistema $\mathrm{z}$ uporabo obstoječih virov, ki so na voljo $\mathrm{v}$ delavnici. Raziskavo bomo razširili tudi na večje proizvodne enote v omenjeni organizaciji. Trenutno raziskujemo planiranje kapacitet za renoviranje tanka T-80 UD.

\section{PODATKI O ČLANKU}

Ključne besede:

Planiranje kapacitet

Vitko razmišljanje

Proizvodnja

Orodjarna

Simulacija

*Kontaktna oseba:

aftab775@yahoo.com

(Haider, A.)

Zgodovina članka:

Prejet 26. februarja 2015

Popravljen 3. oktobra 2015

Sprejet 20. oktobra 2015 


\section{References}

[1] Sernola, T., (2011). How to initiate a capacity planning and management process for a rapid deployment unit of a security services company, Bachelor's thesis, Tampere University of Applied Sciences.

[2] Chen, C.S., Mestry, S., Damodaran, P., Wang, C. (2009). The capacity planning problem in make-to-order enterprises, Mathematical and Computer Modeling, Vol. 50, No. 9-10, 1461-1473, doi: 10.1016/i.mcm.2009. 07.010.

[3] Linné, A, Ekhall, C.J. (2013). Lean capacity planning - planning for maximizing customer value, Master Thesis, department of technology management and economics, Chalmers university of technology, Sweden.

[4] Azizi, A., Kamaruddin, S. (2008). A review on the methods of shop floor capacity planning and scheduling for semiconductor industry, In: Proceedings of $9^{\text {th }}$ Asia Pacific Industrial Engineering \& Management Systems Conference, 2947-2952.

[5] Beauregard, Y., Thomson, V., Bhuiyan, N. (2008) Lean engineering logistics: load leveling of design jobs with capacity considerations, Canadian Aeronautics and Space Journal, Vol 54, No. 2, doi: 10.5589/q08-006.

[6] White, B., Chang, Y., Grabowski, B., Brown, D. (2014). Using lean-based systems engineering to increase capacity in the emergency department, Western Journal of Emergency Medicine: Integrating Emergency Care with Population Health, Vol. 15, No. 7, 770-776, doi: 10.5811/westjem.2014.8.21272.

[7] Swallmeh, E., Tobail, A., Abu-Hamad, W., Gray, J., Arisha, A. (2014). Integrating Simulation Modeling and Value Stream Mapping for Leaner Capacity Planning of an Emergency Department, In: Proceedings of $6^{\text {th }}$ International Conference on Sdvances in System Simulation, 256-262.

[8] Varodhomwathana, T., Sabsomboon, S., Production process design for automotive part with the application of lean manufacturing and computer simulation, In: Proceedings of International conference on Innovative Engineering Technologies, Bangkok, 37-41, doi: 10.15242/IIE.E1214044.

[9] Nik Mohamed, N.M.Z., Ab Rashid, M.F.F., Mohd Rose, A.N., Ting, W.Y. (2015). Production layout improvement for steel fabrication works, Journal of Industrial and Intelligent Information, Vol. 3, No. 2, 133-137. doi: 10.12720/jiii.3.2.133-137.

[10] De Carlo, F., Arleo, M.A., Borgia, O., Tucci, M. (2013). Layout design for a low capacity manufacturing line: A case study, International Journal of Engineering Business Management, Vol. 5, Special Issue on Innovations in Fashion Industry, 1-10, doi: 10.5772/56883.

[11] Nguyen, D.M. (2015). A new application model of lean management in small and medium sized enterprises, International Journal of Simulation Modelling, Vol. 14, No. 2, 289-298, doi: 10.2507/IJSIMM14(2)9.304.

[12] Spellmann, A., Gimarc, R., Gimarc, C. (2008). Green capacity planning: Theory and practice, In: Proceedings of $34^{\text {th }}$ International Computer Measurement Group Conference, USA.

[13] Zijm, W.H.M., Buitenhek, R. (1996) Capacity planning and lead time management, International Journal of Production Economics, Vol. 46-47, 165-179, doi: 10.1016/0925-5273(95)00161-1.

[14] Reid, R.D., Sanders, N.R. (2011). Operations Management, (4th edition), WileyPLUS.

[15] Haider, A.A, Nadeem, A., Rafiq, S.(2014) Multiple objective test suite optimization: A fuzzy logic based approach, Journal of Intelligent and Fuzzy Systems, Vol. 27, No. 2, 863-875, doi: 10.3233/IFS-131045.

[16] Replacing Cycle Time with Flow Time, from http://tameflow.tendon.net/post/56252147592/replacing-cycle-timewith-flow-time, accessed on February 11, 2015.

[17] Haider, A., Jahanzaib, M., Akhtar, K. (2007), Experimental comparison of one piece flow production: A simulation based approach. European Journal of Scientific Research, Vol. 19, No. 1, 97-101.

[18] Haider, A. (2006). Lean scheduling in high variety low volume environment, Master Thesis, University of Engineering and Technology, Taxila, Pakistan.

[19] Kelton, D., Sadowski, R.P., Sadowski, D.A. (2001). Simulation with Arena, McGraw Hill, New York.

[20] Haider, A., Mirza, J. (2015). An implementation of lean scheduling in a job shop environment, Advances in Production Engineering \& Management, Vol. 10, No. 1, 5-17, doi: 10.14743/apem2015.1.188. 